\title{
Research Article \\ Exact Periodic Solutions of the Nonintegrable Kawahara Equation
}

\author{
Ognyan Yordanov Kamenov and Anna P. Angova
}

Department of Applied Mathematics and Informatics, Technical University of Sofia, P.O. Box 384, 1000 Sofia, Bulgaria

Correspondence should be addressed to Ognyan Yordanov Kamenov, okam@abv.bg

Received 26 April 2012; Accepted 19 July 2012

Academic Editors: M. Ehrnström, K. S. Fa, D. Gepner, and W.-H. Steeb

Copyright (C) 2012 O. Y. Kamenov and A. P. Angova. This is an open access article distributed under the Creative Commons Attribution License, which permits unrestricted use, distribution, and reproduction in any medium, provided the original work is properly cited.

In the present paper, we have obtained an exact biperiodic, one-phase solution of the Kawahara evolution equation. Two classes of real periodic waves generated by the biperiodic solution have been analyzed. A modification of the bilinear-transformation method has been applied allowing to provide a single solution of the residual equation derived from the bidifferential reduction of the considered nonintegrable equation. It is shown that the spatial displacements are individual for each separate harmonic of the real periodic solutions.

\section{Introduction}

The nonlinear evolution equation

$$
u_{t}+\alpha u u_{x}+\beta u_{x x x}=\gamma u_{x x x x x}
$$

was introduced by Kawahara [1] as a model describing one-dimensional waves for which the angle between the front and the gradient of the external field tends to the critical angle: $\varphi_{c}=\operatorname{arctg}\left(\sqrt{m_{1} / m_{0}}-\sqrt{m_{0} / m_{1}}\right)$. Here, $\alpha, \beta$, and $\gamma$ are real parameters $(\alpha \neq 0), u=u(x, t)$ is the elevation of dispersion medium, and $m_{0}, m_{1}$ are the ion and electron masses, respectively. Within the zone of the critical angle $\varphi_{c}$, the coefficient in front of $u_{x x x}$ in (1.1) decreases causing disbalance between the nonlinear and dispersion effects, which is balanced by the member with the highest-order derivative. Therefore, three varieties of the dispersion medium are distinguished: medium with positive dispersion $(\beta<0, \gamma<0)$; medium with negative dispersion $(\beta>0, \gamma>0)$ and medium with mixed dispersion $(\beta \gamma<0)$. Yamamoto [2] obtained the same evolution equation (1.1) in describing the dynamics of a nonviscous 
fluid in the vicinity of the critical depth. In this case, dispersion of the lowest order caused by the gravitation is balanced by the one caused by the surface tension.

The Kawahara evolution equation (1.1) is nonintegrable, which can be easily established by applying the modified criterion of Ablowtz and Segur [3]. Although it does not possess any conservation law and $N-(N \geq 2),(1.1)$ has been a subject of research by a number of authors employing both analytic and numerical methods.

Kano and Nakayama [4] have found elliptic solution of (1.1), from which by means of appropriate phase modulations they obtained periodic cnoidal solutions, as well as M-type and W-type solitary waves. Yamamoto and Takizawa [5] have found a stable progressivepulse solution

$$
u(x, t)=\frac{105 \beta^{2}}{169 \gamma \alpha} \sec ^{4}\left[\frac{1}{2} \sqrt{\frac{\beta}{13 \gamma}}\left(x-\frac{36 \beta^{2}}{169 \gamma} t\right)\right]
$$

which actually represents one-soliton impulse that can be generated only in a medium with positive or negative dispersion (i.e., for $\operatorname{sign} \beta=\operatorname{sign} \gamma$ ). In the particular case $\beta=0$, (1.1) is called FKdV (Five Korteweg-de Vries) and has been thoroughly studied numerically and analytically by Boyd [6]. Summarizing the numerical analysis accomplished by a number of authors $[1,2,7]$, we can say that the Kawahara equation has two types of localized solitary waves: compressed and fictitiously scattered corresponding to a medium with negative and positive dispersion, respectively.

Studies on the existence of periodic solutions of (1.1) are considerably fewer. In 1997 Berloff and Howard [8], by applying the singular manifold method adapted for partial differential equations [9], obtained solitary and approximate periodic solutions of the Kawahara equation.

In the present paper, two families of real periodic solutions of the aforementioned equation are obtained. These exact periodic solutions were proved to be dynamically equivalent. A "spatial" version [10] of the bilinear-transformation method of Hirota [11] and Matsuno [12] has been applied to derive them. In its classic form, this analytic model is inapplicable to nonintegrable partial differential equations.

\section{Preliminaries}

We could reduce the Kawahara equation in the so-called normal form if instead of the parameter $\beta$ we put $\varepsilon \beta$, that is, $\beta \rightarrow \varepsilon \beta$, where $\varepsilon= \pm 1, \beta>0$, and assume that $\gamma>0$.

As will be seen below, the last condition does not lead to loss of generality since after the following rescaling:

$$
x \rightarrow \sqrt{\frac{\gamma}{\beta}} x, \quad t \longrightarrow \frac{\gamma}{\beta^{2}} \sqrt{\frac{\gamma}{\beta}} t, \quad u \longrightarrow \frac{\beta^{2}}{\alpha \gamma} u
$$

(1.1) is reduced to its normal form

$$
u_{t}+u u_{x}+\varepsilon u_{x x x}=u_{x x x x x}, \quad \varepsilon= \pm 1 .
$$


Hence, it is easy to realize that for $\varepsilon=+1$, we have a medium with negative dispersion, for $\varepsilon=+1$ along with replacing the variables $u \rightarrow-u, x \rightarrow-x, t \rightarrow-t$, the medium possesses positive dispersion, while for $\varepsilon=-1$, we have a medium with mixed dispersion, that is, we have all available variations of the dispersion medium.

The Kawahara equation (2.2) is invariant with respect to the Galilean transformation $x^{\prime}=x+\lambda u_{0}, t^{\prime}=t$, and $U=u-u_{0}$, where $\lambda=$ const, $u_{0}=$ const, that is, if $U\left(x-v_{0} t\right)$ is a solution of (2.2) with constant phase velocity $v_{0}$, then the function $u(x, t)=u_{0}+U\left[x-\left(v_{0}+\lambda u_{0}\right) t\right]$ is also a solution of the same equation but with increased phase velocity $v_{0} \rightarrow v_{0}+\lambda u_{0}$.

\section{Biperiodic Solution}

We will represent the solution of (2.2) by means of the Hirota-Satsuma [13] transformation

$$
u(x, t)=a+2 \mu(\ln f(x, t))_{x x^{\prime}}
$$

where $a, \mu$ are unknown parameters so far, and $f(x, t)$ is unknown function, but on the assumption periodic and continuously differentiable to seventh order (with respect to $x$ ) in the semi-infinite domain $\Omega=\left\{(x, t) \in R^{2},-\infty<x<\infty, t>0\right\}$. By substituting $u(x, t)$ from (3.1) into (2.2) and employing the bidifferential identities for the Hirota operator (see Appendix A)

$$
D_{t}^{n} D_{x}^{m} \varphi(x, t) \cdot \psi\left(x^{\prime}, t^{\prime}\right)=\left.\left(\frac{\partial}{\partial t}-\frac{\partial}{\partial t^{\prime}}\right)^{n}\left(\frac{\partial}{\partial x}-\frac{\partial}{\partial x^{\prime}}\right)^{m} \varphi(x, t) \psi\left(x^{\prime}, t^{\prime}\right)\right|_{\substack{t=t^{\prime} \\ x=x^{\prime}}}
$$

we will obtain the following bidifferential form of this equation:

$$
\begin{gathered}
f^{4}\left[D_{t} D_{x}+\varepsilon D_{x}^{4}-D_{x}^{6}-8 C\right] f \cdot f+\frac{f^{2}}{2}(\mu-6 \varepsilon)\left(D_{x}^{2} f \cdot f\right)^{2} \\
+D_{x}^{2} f \cdot f\left[15 f^{2}\left(D_{x}^{4} f \cdot f\right)+\alpha f^{4}-30\left(D_{x}^{2} f \cdot f\right)^{2}\right]=0
\end{gathered}
$$

where $C$ is an integration constant assumed to be nonzero. This constant plays a major role in constructing the periodic solutions though it does not have any dynamic features. If in (3.3) we put $\mu=6 \varepsilon$, then a sufficient condition for the function $f(x, t)$ to be its solution is to satisfy the following two equations accordingly:

$$
\begin{gathered}
\left(D_{t} D_{x}+\varepsilon D_{x}^{4}-D_{x}^{6}-4 C\right) f \cdot f=0, \\
a f^{4}+15 f^{2}\left(D_{x}^{4} f \cdot f\right)=30\left(D_{x}^{2} f \cdot f\right)^{2} .
\end{gathered}
$$

The first of these equations is called bidifferential (since its structure is such), and the second one will be called residual. We will search for the solution of (3.4) in the form

$$
f(x, t)=\theta_{3}(\xi, q)=\sum_{n=-\infty}^{\infty} q^{n^{2}} e^{2 i n \xi},
$$


where $\theta_{3}$ is the Jacobi biperiodic function [14] with phase variable $\xi=k x+\omega t+\delta$. It is possible that the parameters $k, \omega, \delta$ are complex and $q=e^{i \pi \tau}$, and $\operatorname{Im} \tau>0$ (i.e., $0<|q|<1$ ) is the perturbation parameter. The function $\theta_{3}(\xi, q)$ has a real period $\pi / k$ and an imaginary one $\tau / k$. We substitute $f(x, t)$ from (3.6) into the bidifferential (3.4). We obtain an infinite system of algebraic equations

$$
\sum_{m=-\infty}^{\infty} F(m) e^{2 i m \xi}=0
$$

where

$$
F_{m}(m)=\sum_{n=-\infty}^{\infty}\left[-4 k \omega(2 n-m)^{2}+16 \varepsilon k^{4}(2 n-m)^{4}-64 k^{6}(2 n-m)^{6}+8 C\right] q^{n^{2}+(n-m)^{2}}
$$

and having $m=0, \pm 1, \pm 2, \ldots$

The bilinear structure of (3.7) makes it possible to apply the index parity principle therein, which means that if in (3.8) we substitute $n \rightarrow n+1$, we will have the following relations:

$$
\begin{aligned}
F(m) & =F(m-2) q^{2(m-1)}=F(m-4) q^{2(2 m-4)} \\
& =\cdots=F(0) q^{m^{2} / 2} \quad \text { if } m \text { is an even number } \\
F(m) & =F(m-2) q^{2(m-1)}=F(m-4) q^{2(2 m-4)} \\
& =\cdots=F(1) q^{\left(m^{2}-1\right) / 2} \quad \text { if } m \text { is an odd number. }
\end{aligned}
$$

Summing up separately the even and odd addends in (3.7), we will obtain the following more compact form of this equation:

$$
F(0) \theta_{3}\left(2 \xi, q^{2}\right)+q^{-1 / 2} F(1) \theta_{2}\left(2 \xi, q^{2}\right)=0,
$$

where $\theta_{2}(z, q)$ is the second Jacobi biperiodic function [14] defined by the equality $\theta_{2}(z, q)=$ $\sum_{n=-\infty}^{\infty} q^{(n+1 / 2)^{2}} e^{i(2 n-1) z}$. Accounting for the linear independence of the functions $\theta_{2}\left(2 \xi, q^{2}\right)$ and $\theta_{3}\left(2 \xi, q^{2}\right)$ in (3.10), it is reduced to two equations: $F(0)=0$ and $F(1)=0$. In the context of equality (3.8) and the functional identities for the $\theta$-functions, given in Appendix $B$, the last two equations are reduced to the following algebraic linear system:

$$
\begin{aligned}
& \left(k \theta_{3}^{\prime}\right) \omega+\left(\frac{\theta_{3}}{q}\right) C=8 \varepsilon k^{4}\left(\theta_{3}^{\prime}+q \theta_{3}^{\prime \prime}\right)-64 k^{6}\left(\theta_{3}^{\prime}+3 q \theta_{3}^{\prime \prime}+q^{2} \theta_{3}^{\prime \prime \prime}\right) \\
& \left(k \theta_{2}^{\prime}\right) \omega+\left(\frac{\theta_{2}}{q}\right) C=8 \varepsilon k^{4}\left(\theta_{2}^{\prime}+q \theta_{2}^{\prime \prime}\right)-64 k^{6}\left(\theta_{2}^{\prime}+3 q \theta_{2}^{\prime \prime}+q^{2} \theta_{2}^{\prime \prime \prime}\right)
\end{aligned}
$$

with respect to $\omega$ and $C$. This system is compatible and definite since $\Delta=(k / q)\left(\theta_{2} \theta_{3}^{\prime}-\theta_{3} \theta_{2}^{\prime}\right)=$ $(k / q) W\left(\theta_{2}, \theta_{3}\right) \neq 0$, where $W$ is the Wronskian determinant, and the wave number is different 
from zero. We have denoted for convenience $\theta_{j}=\theta_{j}\left(0, q^{2}\right), j=2,3$, so that all derivatives of these functions in the system (3.11) are with respect to the parameter $q$. The solution of system (3.11) is as follows:

$$
\begin{aligned}
& \omega=8 \varepsilon k^{3}\left[1+q \frac{W^{\prime}\left(\theta_{2}, \theta_{3}\right)}{W\left(\theta_{2}, \theta_{3}\right)}\right]-\frac{64 k^{5}}{W\left(\theta_{2}, \theta_{3}\right)}\left\{W\left(\theta_{2}, \theta_{3}\right)+3 q W^{\prime}\left(\theta_{2}, \theta_{3}\right)\right. \\
& \left.+q^{2}\left[W^{\prime \prime}\left(\theta_{2}, \theta_{3}\right)-W\left(\theta_{2}^{\prime}, \theta_{3}^{\prime}\right)\right]\right\}, \\
& C=-8 \varepsilon k^{4} q^{2} \frac{W\left(\theta_{2}^{\prime}, \theta_{3}^{\prime}\right)}{W\left(\theta_{2}, \theta_{3}\right)}+\frac{64 k^{6} q^{2}}{W\left(\theta_{2}, \theta_{3}\right)}\left[W\left(\theta_{2}^{\prime}, \theta_{3}^{\prime}\right)+3 q W^{\prime}\left(\theta_{2}^{\prime}, \theta_{3}^{\prime}\right)\right]
\end{aligned}
$$

The residual equation (3.5) does not possess a bilinear structure, which is a typical feature for the nonintegrable partial differential equations. This means that we cannot apply the index parity principle to (3.5), that is, we cannot reduce the infinite system generated by this equation (after substituting $f(x, t)$ with its equal function $\left.\theta_{3}(\xi, t)\right)$ to a system of two equations. Consequently, we will represent the parameter $a$ in the formal series

$$
a=k^{4} \sum_{m=-\infty}^{\infty} a_{m}
$$

by the terms $a_{m}$, which are real parameters unknown at this stage. Substituting (3.6) and (3.14) into the residual equation (3.5), we will obtain the infinite system

$$
\begin{array}{r}
a_{m} \sum_{n=-\infty}^{\infty} q^{2(n-m)^{2}+(2 n-3 m)^{2}}=240 \sum_{n=-\infty}^{\infty}\left[2 n^{2}(3 n-m)^{2}-(2 n-m)^{4}\right] q^{2 n^{2}+(2 n-m)^{2}}, \\
m=0, \pm 1, \pm 2, \ldots
\end{array}
$$

The infinite series on both sides of the system (3.15) are absolutely convergent for $0<|q|<1$. It is easy to deduce that in summation term by term of the equalities from (3.15) for each whole number $m$, the series (3.14) is an absolutely and uniformly convergent series under the same condition for the perturbation parameter. Thus, we obtain that the terms of the functional series (3.14) are determined for every integer $m$ by the formula

$$
a_{m}(q)=\frac{240 \sum_{n=-\infty}^{\infty}\left[2 n^{2}(3 n-m)^{2}-(2 n-m)^{4}\right] q^{2 n^{2}+(2 n-m)^{2}}}{\sum_{n=-\infty}^{\infty} q^{2(n-m)^{2}+(2 n-3 m)^{2}}}, \quad m=0, \pm 1, \pm 2, \ldots
$$

The terms $a_{m}(q)$ of the uniformly convergent series (3.14) will be called spatial displacements. This will be explained further.

And finally in this section, we can make the conclusion that the continuously differentiable function

$$
u(x, t)=k^{4} \sum_{m=-\infty}^{\infty} a_{m}(q)+12 \varepsilon k^{2} \frac{d}{d \xi}\left[\frac{\theta_{3}^{\prime}(\xi, q)}{\theta_{3}(\xi, q)}\right]
$$




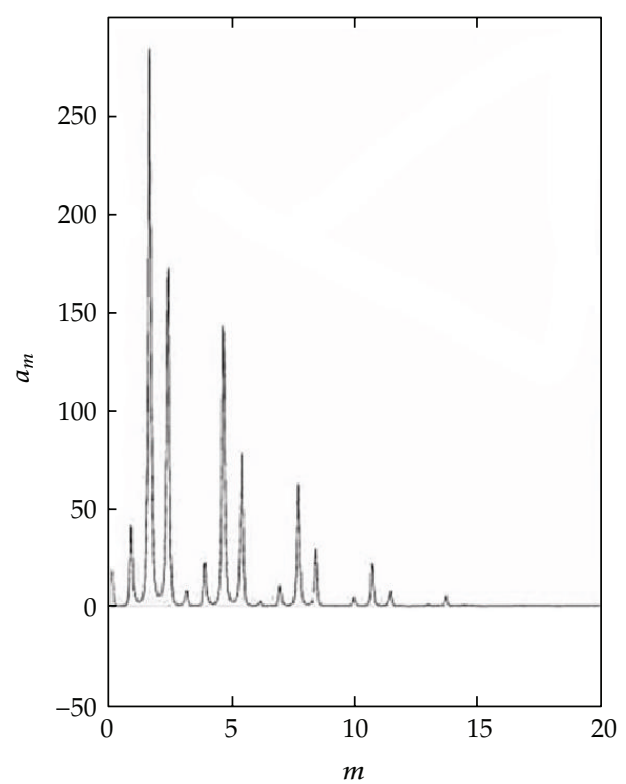

Figure 1: Discrete values of the spatial displacements $a_{m}(q)$ for a mean value of the perturbation parameter $q=e^{-\varepsilon \pi}, \varepsilon=1, k=1$, and $m=0,1,2, \ldots$

is a family of localized biperiodic solutions of the Kawahara equation (2.2). For convenience, in (3.17), we have denoted

$$
\theta_{3}^{\prime}(\xi, q)=\frac{d}{d \xi} \theta_{3}(\xi, q)
$$

Figure 1 shows the discrete values of the spatial displacements $a_{m}(q)$ for a mean value of the perturbation parameter $q=e^{-\varepsilon \pi}, \varepsilon=1, k=1$, and $m=0,1,2, \ldots$ In addition to the foregoing, the function $u(x, t)$, expressed by (3.17), is a solution of the considered equation if the phase velocity $\omega(k, q)$ satisfies the dispersion relation (3.12), and the integration constant $C$ is as in (3.13). The wave number $k \neq 0$ is complex in the general case, but we have to exclude those values for which the constant $C$ is zero, that is,

$$
k^{2} \neq \frac{\varepsilon}{8}\left[1+3 q \frac{W^{\prime}\left(\theta_{2}^{\prime}\left(0, q^{2}\right), \theta_{3}^{\prime}\left(0, q^{2}\right)\right)}{W\left(\theta_{2}^{\prime}\left(0, q^{2}\right), \theta_{3}^{\prime}\left(0, q^{2}\right)\right)}\right]^{-1} .
$$

\section{Analicity Condition and Real Biperiodic Solutions}

The obtained exact biperiodic solution (3.17) of the Kawahara evolution equation (2.2) is generally speaking a rational complex function of the phase variable $\xi=k x+\omega t+\delta$. This function has twofold poles in the lattice of complex points $\xi_{m n}=\pi(m+1 / 2)+i \tau \pi(n+1 / 2)$, $m, n \in Z$, which are zeros of the function $\theta_{3}(\xi, q)$. As we are interested in the physical relevance of the obtained biperiodic solution, we can suitably choose the free parameters $k, q, \delta$, 
so that we can achieve real biperiodic analogues of the solution and at the same time avoid the twofold poles. For this purpose, let us assume that

$$
\tau=i s, \quad s>0, \text { that is, } q=e^{-\pi s}<1 .
$$

Under these conditions, to avoid the poles of the solution $u(x, t)$ at the points $\xi_{m n}$, it is sufficient to limit the phase variable within the horizontal strip

$$
-\pi s<\operatorname{Im}(\xi)<\pi s,
$$

which is actually the analicity condition for the solution. To provide real biperiodic solutions from (3.17), we will consider two possible options for the wave number $k$, under the condition of (3.19).

(i) The wave number $k$ is real. Under this assumption and provided the condition of (4.1) holds, the phase variable $\xi=k x+\omega t+\delta$ is real for real phase shift $\delta$. The logarithmic derivative from (3.17) in this case can be expressed in a Fourier series [14] by the equality

$$
\frac{\theta_{3}^{\prime}(\xi, q)}{\theta_{3}(\xi, q)}=4 \sum_{m=-\infty}^{\infty} \frac{(-1)^{m} q^{m}}{1-q^{2 m}} \sin (2 m \xi)
$$

Thus, from (3.17), we obtain a well-defined, real function which is periodical on the real straight line

$$
u(x, t)=k^{2} \sum_{m=-\infty}^{\infty}\left[k^{2} a_{m}(q)+48 \varepsilon m(-1)^{m} \cos \operatorname{ch}(m \pi s) \cdot \cos (2 m \xi)\right] .
$$

Figure 2 illustrates the forms of the real periodic solution for a real value of the wave number $k,(k=1)$. It can be seen from the last formula that the spatial displacements $a_{m}(q)$ contribute to each separate harmonic of the biperiodic solution.

(ii) The wave number $k$ is imaginary. Let $k \rightarrow i k$, and without loss of generality, we can assume that $k>0$. Under this hypothesis, it is possible to generate real periodic solutions if the phase velocity is an imaginary number and the phase shift is properly chosen. It is obvious from the dispersion equality (3.12) that the phase velocity $\omega$ is an imaginary number (since $\omega(k)$ contains only odd powers of $k$ ). For the phase shift $\delta$, we choose $\delta \rightarrow \delta+\pi \tau$, and hence $i \xi \rightarrow i \xi+\pi \tau$. With this transition, the dispersion relation (3.12) remains the same. Applying the quasiperiodic property for $\theta_{3}$,

$$
\theta_{2}(i \xi, q)=q^{1 / 4} e^{i \xi} \theta_{3}\left(i \xi+\frac{\pi \tau}{2}, q\right)
$$




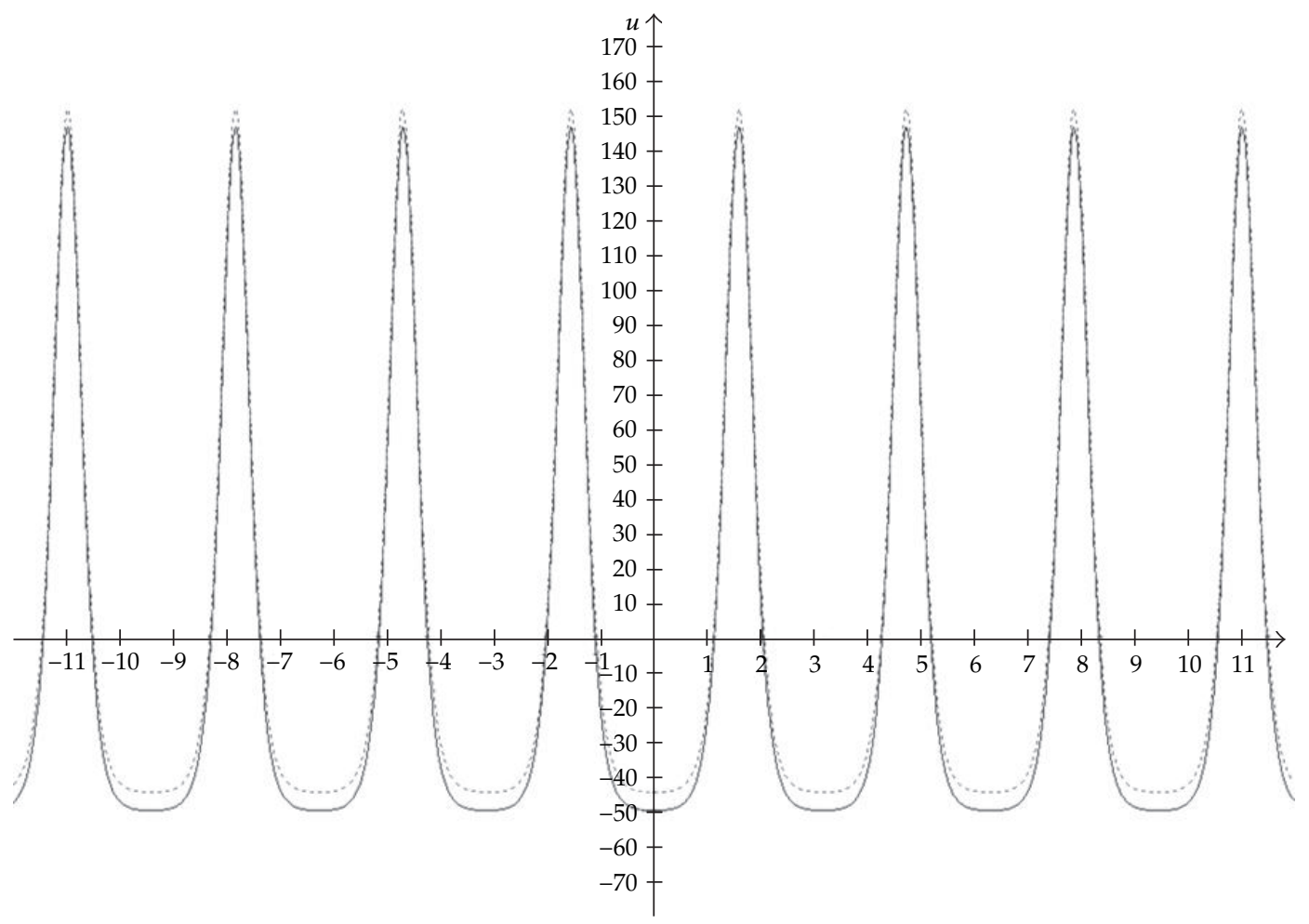

Figure 2: Real sinusoidal periodic solutions for real values of the wave number: $k=1 / 2,1$ and $q=e^{-\pi}$, $\varepsilon=1$.

and from formula (3.17), we have for the logarithmic derivative (see [2])

$$
\frac{d^{2}}{d \xi^{2}} \ln \theta_{2}(i \xi, q)=-\sum_{m=-\infty}^{\infty} \sec h^{2}[i(i \xi-i m \pi s)]
$$

In this case, we also obtain a real periodic function which is well defined in the strip (4.2):

$$
u(x, t)=\sum_{m=-\infty}^{\infty}\left[k^{4} a_{m}(q)+12 \varepsilon k^{2} \sec h^{2}(\xi-m \pi s)\right]
$$

Figure 3 visualizes the periodic solitary-wave profiles of the sec $h^{2}$ type for imaginary values of the wave number $k: i, i / 2, i / 4$, and $q=e^{-\pi}$. Despite their structural differences, the two types of real biperiodic solutions of the Kawahara equation obtained in (4.4) and (4.7) are dynamically equivalent. This comes to confirm the nonlinear superposition principle established by Toda [15] for the KdV evolution equation. In conclusion, let us note that the biperiodic real solutions (4.4) and (4.7) actually describe the dynamics of dispersing waves resulting from the dispersion relation (3.12). Indeed, $\omega^{\prime \prime}(k) \neq 0$, which means that these waves 


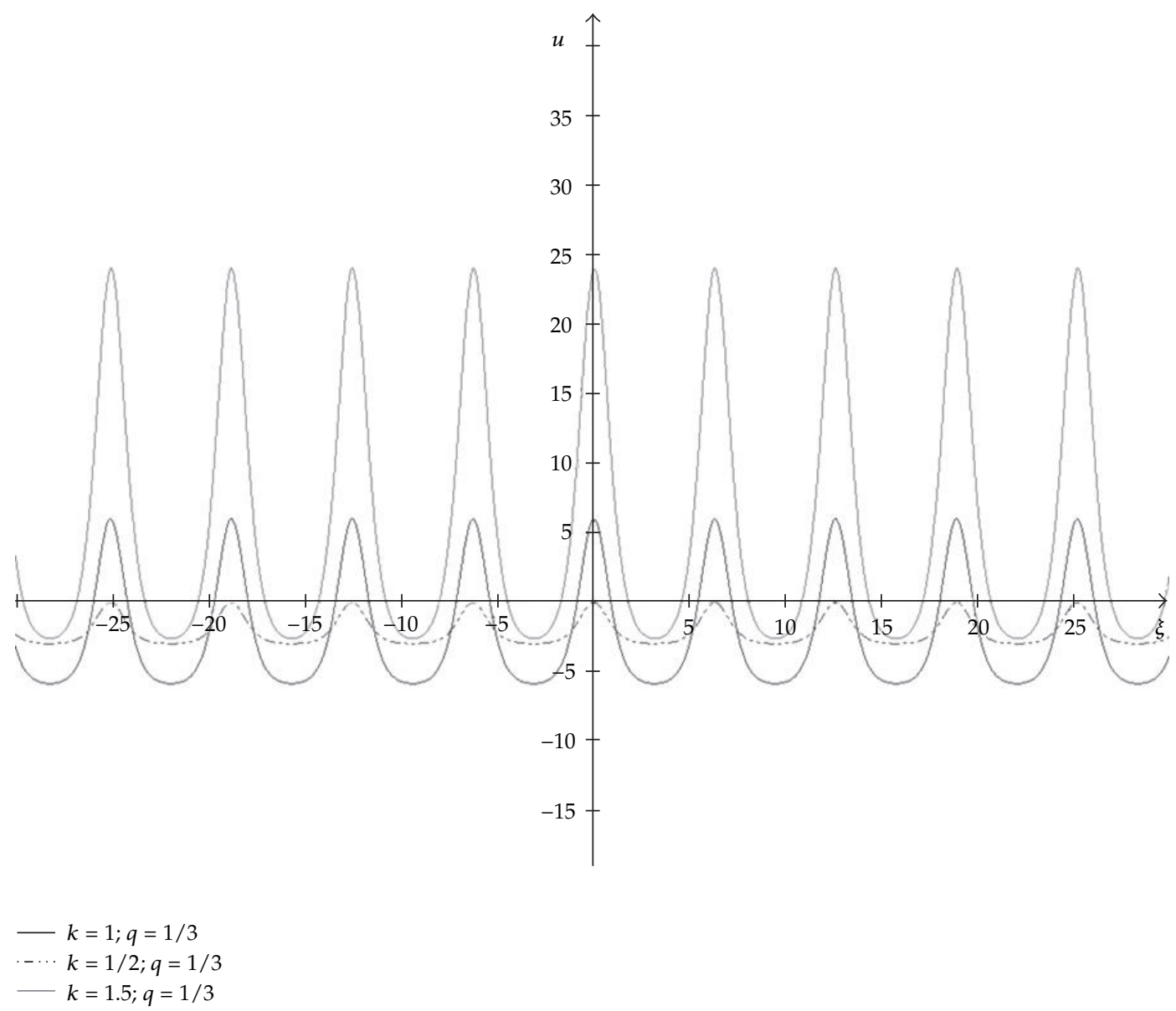

Figure 3: Periodic solitary-wave profiles of sec $h^{2}$ type for imaginary values of the wave number: $k=$ $i, i / 2, i / 4$ and $q=e^{-\pi}$.

are propagating in two directions, as periodically repeating groups traveling with group velocity $V=\omega^{\prime}(k)$.

\section{Conclusions}

Regardless of the purely technical difficulties arising in the application of the bilinear transformation method, it turns out to be a versatile theoretical instrument in analyzing nonintegrable, nonlinear partial differential equations, such as the Kawahara equation (1.1). The major intricacy arises from the residual equation after the bilinear reduction of the initial equation. Harmonization of the bilinear and the residual equation practically means to satisfy an algebraic system with infinite number of equations, but with a finite number of unknowns (usually one or two). The solution of a similar infinite system is feasible if a suitable unknown quantity is presented in the form of a convergent numerical or functional series with unknown terms-a procedure often applied in the Fourier method for linear partial 
differential equations. What is most surprising here is that the terms of these numerical or functional series have unambiguous physical interpretation. They describe spatial displacements individually for each harmonic. For this reason, we called this modification of the bilinear-transformation method, as well in [10], "spatial." Note that for some nonintegrable nonlinear evolution equations, such as the Kuramoto-Sivashinsky equation

$$
u_{t}+u u_{x}+\alpha_{1} u_{x x}+\alpha_{2} u_{x x x}+\alpha_{3} u_{x x x x}=0
$$

spatial displacements are absent. In this case, their role is taken by the "wave" displacements, that is, the wave number is presented as a convergent infinite series.

\section{Appendices}

\section{A. Logarithmic Derivatives Expressed by the Hirota's Bilinear Differential Operators $D_{t}, D_{x}$}

Consider the following:

$$
\begin{aligned}
(\ln \zeta)_{x x} & =\frac{D_{x}^{2} \zeta \cdot \zeta}{2 \zeta^{2}} ; \quad(\ln \zeta)_{t x}=\frac{D_{t} D_{x} \zeta \cdot \zeta}{2 \zeta^{2}} \\
(\ln \zeta)_{x x x x} & =\frac{D_{x}^{4} \zeta \cdot \zeta}{2 \zeta^{2}}-6\left(\frac{D_{x}^{2} \zeta \cdot \zeta}{2 \zeta^{2}}\right)^{2} \\
(\ln \zeta)_{x x x x x x} & =\frac{D_{x}^{6} \zeta \cdot \zeta}{2 \zeta^{2}}-30\left(\frac{D_{x}^{2} \zeta \cdot \zeta}{2 \zeta^{2}}\right)\left(\frac{D_{x}^{4} \zeta \cdot \zeta}{2 \zeta^{2}}\right)+120\left(\frac{D_{x}^{2} \zeta \cdot \zeta}{2 \zeta^{2}}\right)^{3}
\end{aligned}
$$

\section{B. Identities for the Jacobi $\theta$-Functions}

Consider the following:

$$
\begin{array}{cc}
\sum_{n=-\infty}^{\infty} q^{2 n^{2}}=\theta_{3}=\theta_{3}\left(0, q^{2}\right), & \sum_{n=-\infty}^{\infty} q^{n^{2}+(n-1)^{2}}=q^{1 / 2} \theta_{2}=q^{1 / 2} \theta_{2}\left(0, q^{2}\right) \\
\sum_{n=-\infty}^{\infty} n^{2} q^{2 n^{2}}=\frac{q \theta_{3}^{\prime}}{2}, & \sum_{n=-\infty}^{\infty}(2 n-1)^{2} q^{n^{2}+(n-1)^{2}}=2 q^{3 / 2} \theta_{2}^{\prime} \\
\sum_{n=-\infty}^{\infty} n^{4} q^{2 n^{2}}=\frac{q\left(\theta_{3}^{\prime}+q \theta_{3}^{\prime \prime}\right)}{4}, & \sum_{n=-\infty}^{\infty}(2 n-1)^{4} q^{n^{2}+(n-1)^{2}}=4 q^{3 / 2}\left(\theta_{2}^{\prime}+q \theta_{2}^{\prime \prime}\right)
\end{array}
$$




$$
\begin{gathered}
\sum_{n=-\infty}^{\infty} n^{6} q^{2 n^{2}}=\frac{q\left(\theta_{3}^{\prime}+3 q \theta_{3}^{\prime \prime}+q^{2} \theta_{3}^{\prime \prime \prime}\right)}{8} \\
\sum_{n=-\infty}^{\infty}(2 n-1)^{6} q^{n^{2}+(n-1)^{2}}=8 q^{3 / 2}\left(\theta_{2}^{\prime}+3 q \theta_{2}^{\prime \prime}+q^{2} \theta_{2}^{\prime \prime \prime}\right) .
\end{gathered}
$$

\section{Acknowledgment}

The authors would like to thank the Academic Editors for the useful remarks that helped in improving the presentation of the paper.

\section{References}

[1] T. Kawahara, "Oscillatory solitary waves in dispersive media," Journal of the Physical Society of Japan, vol. 33, no. 1, pp. 260-264, 1972.

[2] Y. Yamamoto, "Head-on collision of shallow-water solitary waves near the critical depth where dispersion due to gravity balances with that due to surface tension," Journal of the Physical Society of Japan, vol. 58, no. 12, pp. 4410-4415, 1989.

[3] M. Ablowtz and H. Segur, Solitons and the Inverse Scattering Transform, Cambridge University Press, New York, NY, USA, 2006.

[4] K. Kano and T. Nakayama, "An exact solution of the wave equation: $u_{t}+u u_{x}=u_{x x x x x}$ " Journal of the Physical Society of Japan, vol. 50, no. 2, pp. 361-362, 1981.

[5] Y. Yamamoto and E. Takizawa, "On a solution on non-linear time-evolution equation of fifth order," Journal of the Physical Society of Japan, vol. 50, no. 5, pp. 1421-1422, 1981.

[6] J. P. Boyd, "Solitons from sine waves: analytical and numerical methods for non-integrable solitary and cnoidal waves," Physica D, vol. 21, no. 2-3, pp. 227-246, 1986.

[7] J. K. Hunter and J. Scheurle, "Existence of perturbed solitary wave solutions to a model equation for water waves," Physica D, vol. 32, no. 2, pp. 253-268, 1988.

[8] N. G. Berloff and L. N. Howard, "Solitary and periodic solutions of nonlinear nonintegrable equations," Studies in Applied Mathematics, vol. 99, no. 1, pp. 1-24, 1997.

[9] M. Weiss, M. Tabor, and G. Carneval, "The Painleve property for partial differential equation," Journal of Mathematical Physics, vol. 24, no. 3, pp. 522-526, 1983.

[10] O. Y. Kamenov, "Exact periodic solutions of the sixth-order generalized Boussinesq equation," Journal of Physics A, vol. 42, no. 37, Article ID 375501, 11 pages, 2009.

[11] R. Hirota, "Exact N-soliton solutions of the wave equation of long waves in shallow-water and in nonlinear lattices," Journal of Mathematical Physics, vol. 14, no. 7, pp. 810-814, 1973.

[12] Y. Matsuno, Bilinear Transformation Method, Academic Press, New York, NY, USA, 1984.

[13] R. Hirota and J. Satsumai, "N-soliton solutions of model equations for shallow water waves," Journal of the Physical Society of Japan, vol. 40, no. 2, pp. 611-612, 1976.

[14] E. Janke, F. Emde, and F. Lösch, Tafeln Höherer Funktionen, B.G. Teubner, Stuttgart, Germany, 1960.

[15] M. Toda, Theory of Nonlinear Lattices, Springer, New York, NY, USA, 1979. 


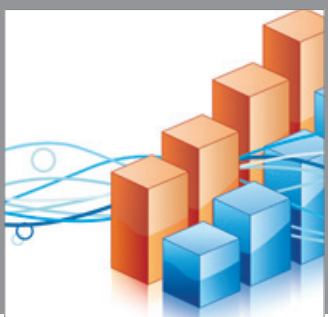

Advances in

Operations Research

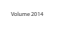

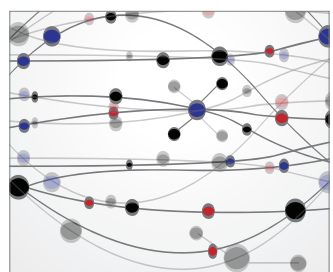

\section{The Scientific} World Journal
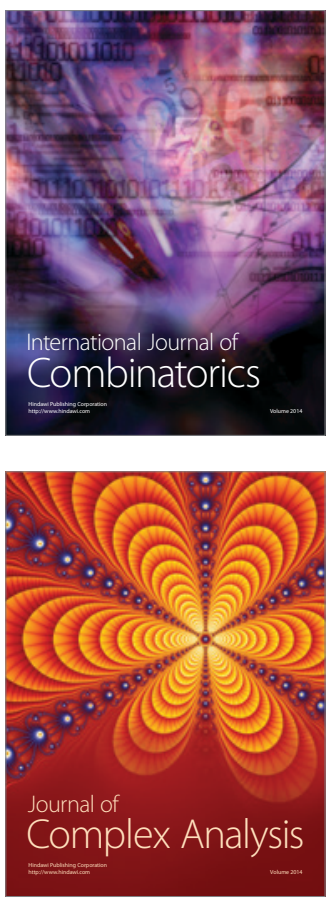

International Journal of

Mathematics and

Mathematical

Sciences
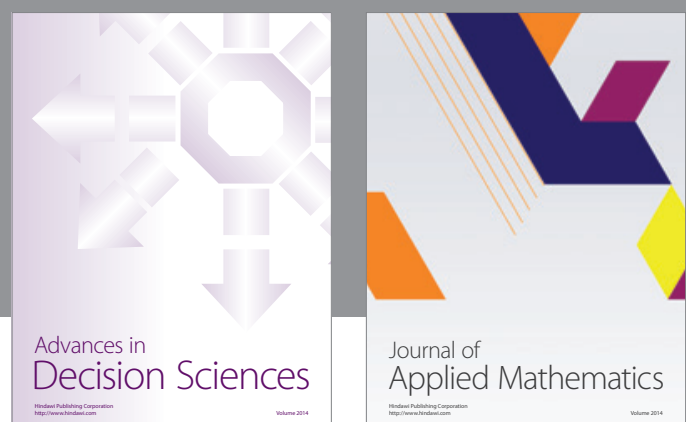

Journal of

Applied Mathematics
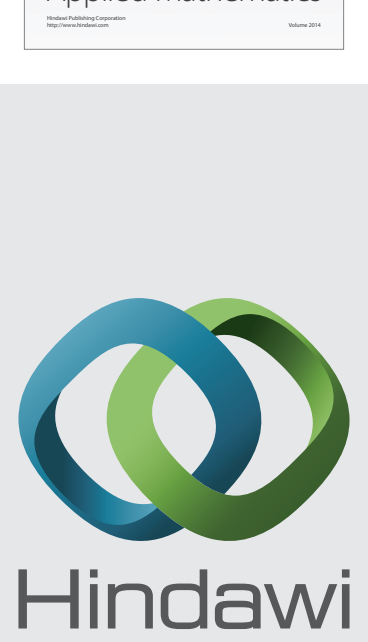

Submit your manuscripts at http://www.hindawi.com
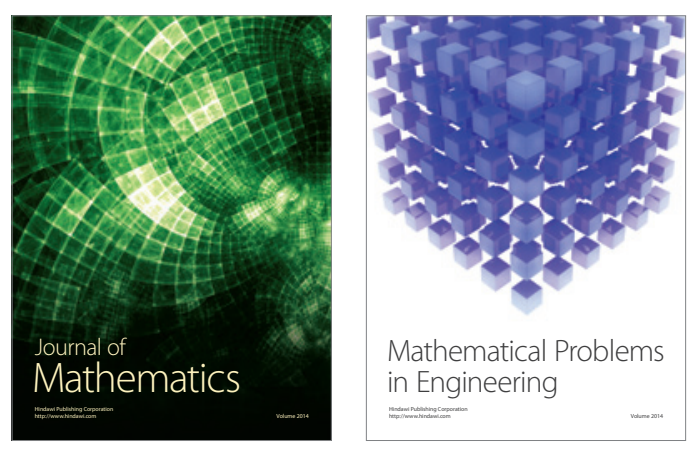

Mathematical Problems in Engineering
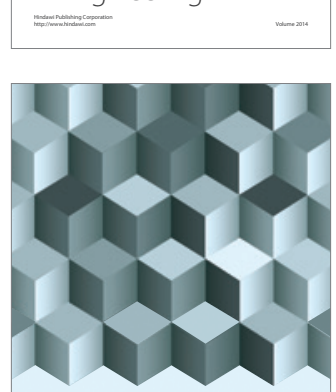

Journal of

Function Spaces
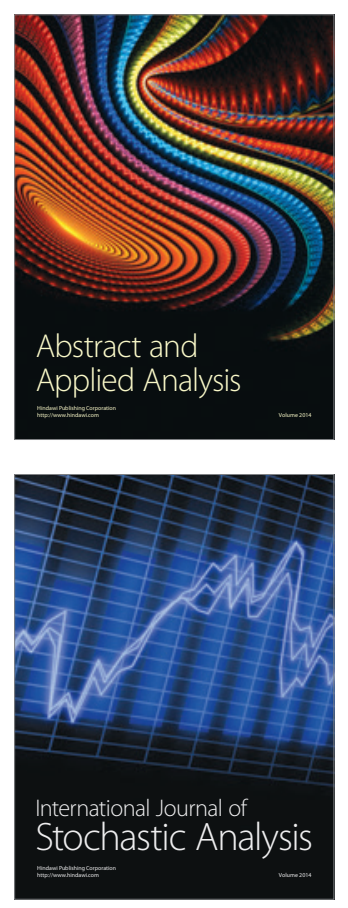

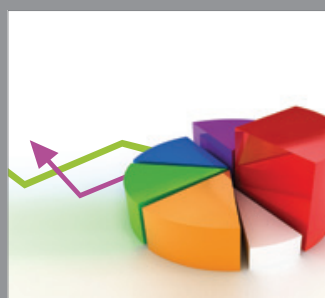

ournal of

Probability and Statistics

Promensencen
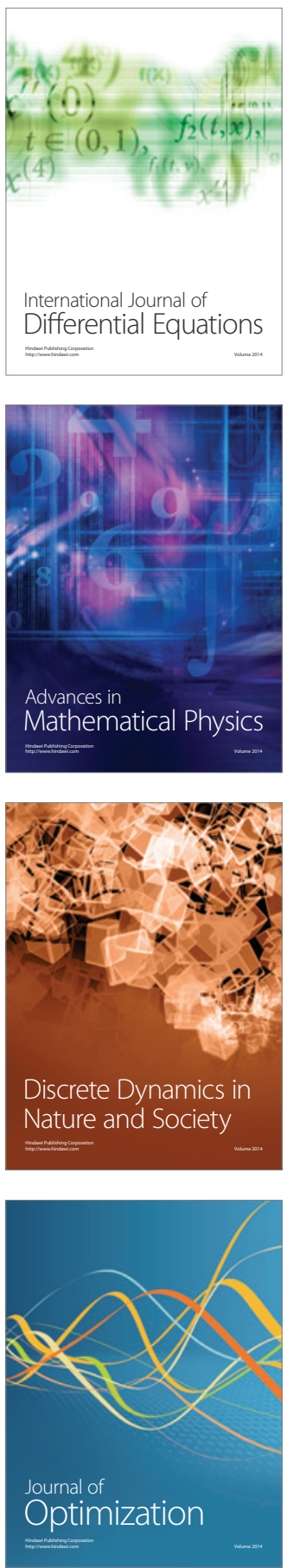